\title{
Paracanoagem e qualidade de vida: concepções de atletas com deficiência motora de Petrolina - PE
}

\section{RESUMO}

O objetivo do presente estudo foi analisar a concepção de atletas de paracanoagem sobre as relações dessa modalidade paralímpica com a qualidade de vida. A amostra foi composta por nove pessoas praticantes de paracanoagem com deficiência motora residentes na região de Petrolina - PE. Os indivíduos participaram de uma entrevista semiestruturada contendo questões referentes à concepção deles próprios acerca dos efeitos da prática desse esporte na qualidade de vida. Posteriormente, as entrevistas foram transcritas e submetidas à análise de dados por meio da Análise de Conteúdo, modalidade Temática. Todos os participantes relataram que a prática da paracanoagem influenciou positivamente aspectos representados em oito categorias: sono, equilíbrio corporal, saúde, força muscular, digestão, autoestima, coordenação motora e respiração. Com isso, concluiu-se que a prática da paracanoagem teve influência positiva na qualidade de vida das pessoas com deficiência motora participantes deste estudo.

PALAVRAS-CHAVE: Paracanoagem; Qualidade de vida; Deficiência motora; Esporte paralímpico; Esporte adaptado
Débora de Souza Araújo Graduada em Educação Física. Universidade Federal do Vale do São Francisco - UNIVASF, Petrolina, Pernambuco, Brasil. deborasouza.araujo@hotmail.com

(ㄱ https://orcid.org/0000-0002-4104-7484

Samuel da Silva Lima Mestrando em Educação Física. Universidade Federal do Vale do São Francisco - UNIVASF,

Petrolina, Pernambuco, Brasil. samuel.lima7@hotmail.com ${ }^{\circ}$ https://orcid.org/0000-0003-4666-1130

Renan Costa Vanali Mestrado Profissional em Saúde da Criança e do Adolescente. Centro Universitário Dr. Leão Sampaio - UNILEÃO, Juazeiro do Norte Ceará, Brasil. ifrenanvanali@gmail.com ${ }^{\circ}$ https://orcid.org/0000-0002-4193-3363

\section{Luiz Augusto Mazine dos Santos}

Pós Graduado em Fisioterapia Ortopédica e Traumatológica. Instituto Meninos do Lago IMEL, Foz do Iguaçu, Paraná, Brasil. fisioguto@hotmail.com

ำ https://orcid.org/0000-0002-4824-4173

Leonardo Gasques Trevisan Costa Doutorado em Educação Física. Universidade Federal do Vale do São Francisco - UNIVASF, Petrolina, Pernambuco, Brasil. leonardo.gasques@univasf.edu.br https://orcid.org/0000-0001-7711-205X 


\title{
Paracanoe and quality of life: concepts of athlets with motor disabilities in Petrolina - PE
}

\begin{abstract}
The objective of the present study was to analyze what perception paracanoe athlets have about the relations of this paralympic sport with life quality. The sample consisted of nine people with motor disability who live in the region of Petrolina - PE, Brazil. The subjects participated in a semistructured interview containing questions regarding their conception about the effects of the practice of this sport on life quality. Subsequently, the interviews were transcribed and subjected to data analysis through Content Analysis, Thematic modality. All participants reported that the practice of paracanoe positively influenced aspects represented in eight categories: sleep, body balance, health, muscle strength, digestion, self-esteem, motor coordination and breathing. Thus, it was concluded that the paracanoe practice had a positive influence on the life quality of the people with motor disabilities in this study.
\end{abstract}

KEYWORDS: Paracanoe; Quality of life; Motor disability; Paralympic sport; Adapted sport

Paracanotaje y calidad de vida: concepciones de atletas con discapacidad motora en Petrolina - PE

\section{RESUMEN}

El objetivo del presente estudio fue analizar el concepto de los atletas paracanotaje sobre las relaciones de este deporte paralímpico con la calidad de vida. La muestra consistió en nueve personas con discapacidad motora que viven en la región de Petrolina - PE. Los sujetos participaron en una entrevista semiestructurada que contenía preguntas sobre su concepción sobre los efectos de la práctica de este deporte en la calidad de vida. Posteriormente, las entrevistas fueron transcritas y sometidas a análisis de datos a través de Análisis de Contenido, modalidad temática. Todos los participantes informaron que la práctica del paracanotaje influyó positivamente en aspectos representados en ocho categorías: sueño, equilibrio corporal, salud, fuerza muscular, digestión, autoestima, coordinación motora y respiración. Por lo tanto, se concluyó que la práctica del paracanotaje tuvo una influencia positiva en la calidad de vida de las personas con discapacidad motora en este estudio.

PALABRAS-CLAVE: Paracanotaje; Calidad de vida; Discapacidad motora; Deporte paralímpico; Deporte adaptado 


\section{INTRODUÇÃO}

A qualidade de vida pode ser entendida como uma concepção subjetiva que abrange parâmetros das áreas de saúde, arquitetura, urbanismo, lazer, gastronomia, esportes, educação, meio ambiente, segurança pública e privada, entretenimento, novas tecnologias e tudo o que inclui o ser humano, sua cultura e seu meio (COLVER, 2008; ALMEIDA, GUTIERREZ, MARQUES, 2012). É, portanto, a percepção do indivíduo referente à sua posição na vida no contexto da cultura e do sistema de valores nos quais ele vive e em relação aos seus objetivos, expectativas, padrões e preocupações (THE WHOQOL GROUP, 1995).

Dentre os fatores que podem influenciar a qualidade de vida dos sujeitos, a prática de atividade física é considerada como um fator positivo, pois atua na prevenção de patologias, assim como na manutenção e na recuperação da saúde de diversas populações (FERREIRA et al., 2015; HÄFELE et al., 2017). Em relação às pessoas com deficiência, programas de atividades físicas possibilitam uma melhora no bem-estar e na qualidade de vida (DIAZ et al., 2019; KAPSAL et al., 2019) devido aos benefícios relacionados ao condicionamento físico (MANNS et al., 1999), às alterações metabólicas e cardiovasculares (SCHREIBER et al., 2018), à reabilitação, à inclusão social (COSTA E SILVA et al., 2013), à valorização pessoal, à prevenção de enfermidades secundárias e à melhora da autoconfiança para a realização de atividades diárias (BRAZUNA; CASTRO, 2001).

O esporte adaptado pode ser uma vivência que, além do alto rendimento, pode colaborar com a inserção a novas práticas sociais e alterar o seu entendimento de qualidade de vida, uma vez que oportuniza para as pessoas com deficiência um novo horizonte e novas perspectivas (CASTRO, 2005; YONG-SEOK et al., 2018), contribuindo com a reabilitação física, psicológica e social, bem como melhorando, de forma geral, a aptidão física, os ganhos de independência e a autoconfiança para a realização de atividades da vida diária; ademais, contribui para uma melhora do autoconceito e da autoestima dos praticantes (CARDOSO, 2011).

A paracanoagem, enquanto modalidade paralímpica, é um esporte náutico praticado em canoa ou caiaque destinado para pessoas com deficiência motora e está presente nos jogos paralímpicos desde 2016, com as provas de velocidade de 200 metros (INTERNATIONAL CANOE FEDERATION, 2015). Assim, a paracanoagem requer equilíbrio na posição sentada, força muscular e potência cardiorrespiratória (AROL \& EROĞLU, 2018), podendo promover benefícios nos aspectos físicos, motores, psicológicos e sociais (BJERKEFORS; THORSTENSSON, 2006). 
A modalidade contribui com a autonomia, oportuniza um estilo de vida mais saudável (ALBARELLO, 2014) e proporciona ganhos nas capacidades físicas de força, potência, resistência e controle postural (MICHAEL et al., 2008; CASTRO e CARNEIRO, 2010). Desse modo, sugere-se que a prática desse esporte adaptado possua uma influência positiva na qualidade de vida de seus praticantes; entretanto, não foram encontrados estudos que investigassem os efeitos da paracanoagem na qualidade de vida. Nesse sentido, o objetivo da presente pesquisa foi analisar a concepção de atletas de paracanoagem sobre as relações dessa modalidade paralímpica com a qualidade de vida.

\section{MÉTODOS}

Este estudo caracteriza-se como descritivo, transversal e qualitativo, pois buscou descrever as características de um determinado fenômeno que possa ocorrer em uma população ou amostra, sem realizar nenhuma intervenção e por meio de descrições, interpretações e comparações, sem analisar os aspectos numéricos em relação às regras matemáticas e estatísticas (FONTELLES, 2009).

Em relação aos aspectos éticos, obedeceu às diretrizes e normas que regulamentam a pesquisa com seres humanos (lei 196/96) e foi aprovado pelo comitê de ética da Universidade Federal do Vale do São Francisco - Univasf (parecer n. 3.892.500). Todos os participantes consentiram em participar da pesquisa por meio da assinatura do termo de consentimento livre e esclarecido (TCLE).

A pesquisa foi realizada no Iate Clube de Petrolina - Pernambuco, local onde ocorrem as práticas de paracanoagem da presente amostra.

\section{Amostra}

A amostra foi composta por nove pessoas com deficiência motora, sendo oito homens e uma mulher, com faixa etária variando entre 24 e 43 anos, residentes na região de Petrolina - PE e Juazeiro - BA, matriculados no projeto de extensão de paracanoagem ofertado pelo colegiado de Educação Física da Univasf.

Para a participação no estudo, foram estabelecidos como critérios de inclusão apresentar o laudo médico atestando possuir deficiência motora, possuir idade mínima de 18 anos, ser praticante de paracanoagem por pelo menos quatro meses e dispor da assinatura do termo de consentimento livre e esclarecido. Foram adotados como critérios de exclusão a não assinatura inicial do Termo de 
Consentimento Livre e Esclarecido e a retirada voluntária do TCLE a qualquer momento da pesquisa.

\section{Coleta de dados}

A entrevista é uma maneira de coletar dados que, por sua vez, podem ser transcritos para texto, caso seja necessário, e codificados, podendo essa codificação ocorrer em dois níveis: primeiro de significados e categorias, e segundo de temas e relações entre conceitos (SAMPIERI et al., 2013).

Foi utilizado um roteiro de entrevista semiestruturada o qual envolvia sete questões que abordavam o tempo de prática na paracanagem e as concepção da amostra sobre a influência desse esporte na qualidade de vida geral e em componentes específicos relacionados à qualidade de vida (capacidades físicas, atividades da vida diária e sono). Para auxiliar na transcrição e na análise dos dados, as entrevistas foram gravadas por meio de um gravador de voz instalado em aparelho celular (Samsung J7 Prime).

Para a coleta de dados, a pesquisadora responsável convidava individualmente os sujeitos para um local reservado no Iate Clube de Petrolina, que oferecia sigilo e conforto aos participantes. Inicialmente, foi apresentado aos voluntários o objetivo do estudo e o TCLE. Após aceitarem participar, eram iniciadas as entrevistas.

\section{Análise dos dados}

Nesta pesquisa, as entrevistas foram transcritas e, após a transcrição, foram submetidas à análise de dados por meio da Análise de Conteúdo (BARDIN, 2011), sendo que a leitura flutuante e a análise temática foram os recursos recortados do método utilizados neste estudo.

\section{RESULTADOS E DISCUSSÕES}

Participaram deste estudo nove pessoas, sendo oito do sexo masculino e somente uma do sexo feminino. Entre os participantes, a maioria adquiriu a deficiência ao longo da vida, enquanto somente dois possuíam deficiência motora congênita. Os entrevistados possuíam entre 24 e 43 anos de idade e 1 a 43 anos de deficiência. Com relação ao tempo de prática da paracanoagem, todos os atletas treinavam há pelo menos 4 meses. 
Segue, na tabela 1, uma descrição detalhada a respeito de cada participante, levando em consideração a sua idade, o tempo e a etiologia da deficiência, bem como o tempo de prática do esporte.

Tabela 1. Caracterização da amostra do estudo em relação à classificação da deficiência, à classificação funcional, à faixa etária, ao tempo de deficiência e à experiência com a paracanoagem.

\begin{tabular}{|c|c|c|c|c|c|}
\hline Sujeito & Deficiência & $\begin{array}{l}\text { Classificação } \\
\text { funcional }\end{array}$ & Faixa etária & $\begin{array}{c}\text { Tempo de } \\
\text { deficiência } \\
\text { (anos) }\end{array}$ & $\begin{array}{c}\text { Tempo de } \\
\text { prática } \\
\text { (meses) }\end{array}$ \\
\hline 01 & Poliomielite & KL3 & 43 & 42 & 6 \\
\hline 02 & $\begin{array}{l}\text { Amputação } \\
\text { transfemoral } \\
\text { esquerda }\end{array}$ & KL3 & 24 & 1 & 6 \\
\hline 03 & Lesão medular & KL1 & 27 & 14 & 7 \\
\hline 04 & $\begin{array}{l}\text { Alteração } \\
\text { Ortopédica no } \\
\text { Joelho direto }\end{array}$ & - & 33 & 10 & 7 \\
\hline 05 & $\begin{array}{c}\text { Deficiência } \\
\text { congênita nos } \\
\text { membros } \\
\text { inferiores }\end{array}$ & KL3 & 38 & 38 & 14 \\
\hline 06 & Poliomielite & KL2 & 34 & 33 & 4 \\
\hline 07 & Poliomielite & KL3 & 43 & 43 & 24 \\
\hline 08 & Lesão medular & KL3 & 38 & 14 & 6 \\
\hline 09 & $\begin{array}{l}\text { Paralisia } \\
\text { Cerebral }\end{array}$ & - & 31 & 31 & 24 \\
\hline
\end{tabular}

Fonte: Autoria própria

Em relação à maior prevalência do sexo masculino na amostra, a deficiência motora apresenta incidência no sexo masculino do que no feminino. Um estudo realizado por Campos et al. (2008) analisou 100 pacientes com traumatismo na coluna vertebral e descobriu que $86 \%$ desses pacientes eram do sexo masculino, predominantemente jovens.

Porém, não se deve deixar de mencionar que diversos fatores socioculturais tornam o acesso das mulheres às práticas esportivas em geral, sejam elas adaptadas ou não, mais restrito. Pode-se citar como exemplo de agente complicador o fato de que, até 1975, era vedado às mulheres praticar “desportos incompatíveis com as condições de sua natureza" (ADELMAN, 2003, p. 447) e, de acordo com o imaginário de feminilidade da época, pode-se afirmar que a canoagem e a 
paracanoagem certamente não eram consideradas compatíveis com essa "natureza feminina" (OLIVEIRA; CHEREM; TUBINO, 2008).

Levantamentos como os de Carmona (2015) e Tonon (2016) demonstram que, além desses fatores já descritos, as mulheres com deficiência enfrentam outras barreiras para ingressar e se manterem no esporte brasileiro, como: falta de incentivo, baixas condições de treinamento, dificuldade de adaptação, pouco reconhecimento, preconceito, entre outros. No entanto, esta é uma visão que tem se alterado gradativamente no decorrer da evolução do esporte, mas ainda são necessários estudos e incentivos neste sentido.

No que se refere à percepção dos participantes com relação às mudanças em sua qualidade de vida após a prática da paracanoagem, foi realizado o seguinte questionamento: "Na sua percepção, a prática da paracanoagem apresentou algum tipo de influência na sua qualidade de vida? De que forma?". Todos os entrevistados responderam que a prática do esporte teve influência positiva em suas vidas.

\footnotetext{
"Sim, não tem como não influenciar, tanto na sua autoestima, que você acaba ficando com ela elevada, como no desafio, desafio de você se equilibrar no barco, desafio de você vencer a cada dia, de você melhorar, então, assim, ela influencia muito." (Sujeito seis).

"Muito, influenciou em questão de ganhar mais equilibrio, ganhar mais controle do tronco e mais força nos braços, porque a gente trabalha muito os braços e o tronco, e cada dia a tendência é melhorar cada vez mais a qualidade de vida da gente." (Sujeito oito).

"Influenciou, sim. Do primeiro dia que eu entrei num caiaque, senti uma liberdade, achei bacana; paracanoagem, amei mesmo, senti liberto, livre, de novo." (Sujeito nove).
}

Corroborando esta constatação, um estudo realizado por Comerlato (2003) demonstrou que a canoagem proporciona significativo aumento da independência, maior compreensão da deficiência, maior abertura para diálogos sobre seu quadro, diminuição do sentimento de autopiedade e rejeição, sentimento de reconhecimento, respeito e orgulho perante a sociedade, resultando em uma mudança positiva na forma de compreender a vida.

Por meio da análise das entrevistas da presente amostra, foi possível destacar oito categorias de benefícios que o esporte os proporcionou, as quais são apresentadas na tabela 2.

Tabela 2. Concepção dos participantes do estudo sobre a influência da prática da paracanoagem na qualidade de vida.

\begin{tabular}{ccc}
\hline Categorias & Frequência (n) & Prevalência (\%) \\
\hline Sono & 2 & 22,2
\end{tabular}


Equilíbrio corporal

\begin{tabular}{ccc} 
Saúde & 2 & 22,2 \\
\hline Força muscular & 4 & 44,4 \\
\hline Digestão & 1 & 11,1 \\
\hline Autoestima & 1 & 11,1 \\
\hline Coordenação motora & 1 & 11,1 \\
Respiração & 1 & 11,1 \\
\hline
\end{tabular}

Fonte: Autoria própria

De acordo com a tabela 2, o conjunto de benefícios que os participantes apontaram como resultantes da prática da paracanoagem abrange o sono, o equilíbrio corporal, a força muscular, a digestão, a autoestima, a coordenação motora e a respiração. Também foi mencionada a saúde, porém, esta nada mais é do que o agrupamento de todos esses outros quesitos.

Dos fatores citados pela amostra, ao serem indagados sobre a melhoria do equilíbrio corporal, os entrevistados responderam:

"Sim... situação de equilíbrio... uma delas ajudou até... pra você ter a noção, soltei a muleta. Eu iniciei, tava usando as duas, passei pra uma, agora tô sem nenhuma. Melhorou muito a questão de estabilidade, controle." (Sujeito quatro).

"A principal diferença que eu senti foi na postura mesmo. Porque lembra que na outra cadeira tinha um encosto que eu falei que usava ele porque minha coluna ficava doendo? Depois que eu comecei a usar essa cadeira nova que eu to usando sem, não tá mais doendo a coluna, eu acho que é por causa da minha postura, que melhorou." (Sujeito três).

Segundo Milosevic et al. (2017), isto se deve ao fato de que os caiaques de velocidade apresentam como uma das principais características a instabilidade, o que obriga os atletas a realizarem ajustes de controle corporal durante a execução dos movimentos, resultando em notáveis melhoras no equilíbrio de seus praticantes.

Além disso, para Arol e Eroğlu (2018), em razão da instabilidade da superfície do caiaque no meio líquido, o equilíbrio em posição sentada viabiliza estímulos sensório-motores que se associam ao equilíbrio em todos os demais movimentos corporais.

Bjerkefors et al. (2007) analisaram os efeitos do treinamento no caiaque ergômetro no controle postural de pessoas com lesão medular. Foram avaliados 10 sujeitos de ambos os sexos por meio de uma plataforma optoeletrônico de análise de movimento Selspot II (Selcom, Partille, Suécia); a intervenção foi composta por 30 sessões de treinamento por um período de 10 semanas e 
os resultados demonstraram um menor deslocamento nas posições anteroposterior e médio lateral após a intervenção. Com isso, os autores concluem que o treinamento em caiaque ergômetro melhorou o controle postural da amostra.

Quando questionados no que diz respeito à força muscular, quatro participantes relataram sentirem alterações positivas, como ganho de força de grupos musculares específicos, que facilitam afazeres diários, como as transferências da cadeira de rodas.

\begin{abstract}
"Sim, também. A gente percebeu que a paracanoagem desenvolve alguns músculos especificos: a questão do peitoral, a questão dos biceps, dos tríceps; então, assim, costas trabalha bastante, além de fortalecer a cintura do abdômen, que é onde sustenta o corpo." (Sujeito um).

"A força, eu senti que deu uma melhorada na transferência; melhoram os braços e, porque a gente vai melhorando a qualidade de vida, para a gente se transferir para a gente fazer as coisas e a força nos braços vai se fortalecendo a cada dia." (Sujeito oito).
\end{abstract}

Taylor e Mc Gruder (1996) corroboram esta afirmação com sua investigação qualitativa sobre praticantes tetraplégicos de canoagem no mar. Esses autores concluíram que, além dos benefícios previamente citados, como o controle postural, o equilíbrio e a rotação de tronco, este esporte também propicia estímulo motor de movimento e acréscimo da força e da resistência muscular. Corroborando com este estudo, Zoppi et al. (2014), com o objetivo de determinar as melhorias fisiológicas e de desempenho dos remadores paralímpicos brasileiros durante uma temporada de treinamento de 32 semanas, demostraram uma melhora na composição corporal, força muscular, capacidade anaeróbia e desempenho do exercício de remadores adaptáveis do sexo masculino com deficiências motoras.

Skucas e Pokvytyte (2018) examinaram os efeitos de doze semanas de treinamento, combinados de força em terra e de natação de resistência para aumentar a força da parte superior do corpo em dezesseis sujeitos, sendo possível observar a melhora nos parâmetros de força da amostra.

A melhora da qualidade do sono também foi um fator relevante percebido por dois dos participantes, melhora esta descrita da seguinte forma:

\footnotetext{
"Agora eu durmo mais, acordo mais disposto; antes eu não acordava tão disposto como hoje, depois que eu comecei a praticar o esporte da paracanoagem... melhorou, durmo bem; no horário certo, dá aquele sono mesmo." (Sujeito sete).

"Cansaço, um cansaço a mais, mas é um cansaço sadio, sabe? Você chega e toma um banho; alimentação também tem que mudar, porque não vai comer muito à noite, ai deu uma melhorada, eu estou dormindo melhor. Melhorou e muito." (Sujeito oito).
} 
O sono, de acordo com Halson (2014), é um processo natural e biológico imprescindível para a regeneração física e cognitiva dos atletas após os treinamentos e competições. Para indivíduos com lesão medular, uma atividade proporcional a uma caminhada gera um gasto energético três vezes maior do que o experimentado por uma pessoa que não possui lesão na medula espinhal (NOREAU et. al., 1993). Esse maior gasto de energia pode justificar o cansaço e, consequentemente, melhora a qualidade do sono (MARTINS; MELLO; TUFIK, 2001).

Uma revisão literária realizada por Ropke et al. (2018), que analisaram o efeito da atividade física na qualidade do sono e na qualidade de vida, aponta que há evidências da melhora na percepção subjetiva e objetiva na qualidade do sono, sendo um ótimo tratamento terapêutico isolado ou coadjuvante para o distúrbio do sono.

No que tange à coordenação motora e à respiração, um participante relatou que a paracanoagem proporcionou melhoria nesses quesitos.

"Sim. Assim... em respiração, em musculação, coordenação motora e, assim, na qualidade de vida.É... a respiração mudou muito. Não tenho o cansaço como tinha antigamente." (Sujeito sete).

Conforme estudo realizado por Jacobs et al. (2001), em que se avaliou o impacto de 12 semanas de treinamento em circuito na capacidade cardiorrespiratória e força muscular em indivíduos com paraplegia, foi possível verificar aumentos significativos no consumo máximo de oxigênio e na potência de pico durante a realização do teste em ergômetro de braço. Corroborando com esses resultados, Flores et al. (2013) analisaram a influência do treinamento de rúgbi em cadeira de rodas nos aspectos fisiológicos e na capacidade funcional dos indivíduos com lesão medular, observando que houve melhora nos valores referentes à variável fisiológica volume de oxigênio máximo após o período de treinamento. Ainda no estudo de Kim et al. (2015), com o objetivo de determinar o efeito de seis semanas de exercício em uma hand-bike quanto aos níveis de insulina, em jejum, e quanto ao perfil cardiometabólico, músculo-esquelético, bem como os níveis de aptidão cardiopulmonar em pessoas com lesão medular, os autores verificaram que, depois do período de intervenção, o volume de oxigênio pico dos sujeitos apresentaram melhora significativa.

A melhoria da autoestima foi observada por um dos participantes, quando apontado que:

"Sim, não tem como não influenciar, tanto na sua autoestima, que você acaba ficando com ela elevada... a questão da autoestima é muito importante, pra qualquer pessoa, porque você, com uma autoestima elevada, você tem mais vontade de seguir em frente..." (Sujeito seis). 
O crescimento da autoestima, na verdade, é um resultado da união de todos os demais quesitos, já que, como conclui o já mencionado estudo de Ropke et. al. (2017), a atividade física melhora a qualidade de vida, isto é, ocorre o aumento do equilíbrio, da qualidade do sono e da força muscular, além de uma maior facilidade de digestão e do aperfeiçoamento da coordenação motora e da respiração, proporcionando uma melhora considerável na saúde do indivíduo; e isso, por si só, já gera um aumento da qualidade de vida das pessoas com deficiência motora. Quando a isso se acrescentam novas experiências, aprendizados e amizades, consequentemente se tem também um aumento na autoestima desse atleta (MATHERI; FRANTZ, 2009).

De acordo com Gorgatti e Costa (2005), o esporte adaptado melhora a aptidão física, a independência e a autoconfiança de pessoas com deficiência motora para a realização de tarefas diárias, além de impulsionar o autoconceito e a autoestima. Os autores ainda afirmam que, mais do que terapia, o esporte, para essa população, caminha para o alto rendimento. O índice geral técnico dos atletas impressiona o público e, com frequência, também os estudiosos da área da atividade física. Pode-se considerar que o esporte adaptado produz consequências positivas no indivíduo que o pratica, não somente a nível fisiológico, mas social também, visto que a atividade proporciona ao praticante maior funcionalidade, o que acarreta em ganho de independência e interação social (EPHIPHANIO et al., 2018).

É importante ressaltar que os eventos nacionais e internacionais de paracanoagem são realizados, em sua maioria, concomitantemente com os eventos da Canoagem velocidade, o que também coloca essa atividade como uma modalidade inclusiva, pois, segundo Marques (1997), o esporte adaptado tem um potencial de inclusão social, sendo contrário às práticas de desvantagem, permitindo, assim, as pessoas com deficiência transporem barreiras restritivas ou impeditivas da sociedade. Para Celestino \& Pereira (2015), o esporte adaptado tem ganhado notoriedade como um agente importante da inclusão e da socialização. Uma das formas de manifestação do esporte adaptado é por meio do esporte paralímpico que, segundo Goodwin et al. (2009), pode ser um agente facilitador para a inclusão social, pois dá a possibilidade do atleta estar em um grupo de pessoas nas mesmas condições e ter seus feitos valorizados não pela superação da deficiência em si, mas por critérios esportivos.

No que se refere à saúde, dois participantes responderam que a paracanoagem favorece esse aspecto em suas vidas.

"Sim, é... melhoria postural, ganho de massa muscular, saúde, né?! Também melhorou." (Sujeito dois). 
Como já enfatizado anteriormente, o termo saúde é muito abrangente e abarca todas as categorias já analisadas. Todas as melhorias relatadas permitem que a saúde em geral evolua. É por este motivo que pessoas com deficiência procuram fazer algum esporte: no intuito de melhorar a saúde. Neste sentido, um estudo de Buffart et al. (2009), realizado com 16 jovens com deficiência motora da Holanda, destacou que os principais motivos para a prática da atividade física são a diversão, a interação e o apoio social, além da melhoria da saúde e da aptidão física. Nahas (2006) afirma que as atividades físicas e desportivas regulares podem reduzir os sintomas de ansiedade e depressão, promover a socialização e aumentar os níveis e o bem-estar geral das pessoas com deficiência.

\section{CONSIDERAÇÕES FINAIS}

Por meio da análise da concepção dos atletas da paracanoagem sobre a influência dessa modalidade na qualidade de vida, conclui-se que existe uma relação positiva entre essa prática paralímpica com os componentes da qualidade de vida analisados no presente estudo (sono, equilíbrio corporal, saúde, força muscular, digestão, autoestima, coordenação motora e respiração), demonstrando que a paracanoagem pode ser utilizada como ferramenta para promover a qualidade de vida de pessoas com deficiência motora.

Os resultados do presente estudo podem ser utilizados como um meio de apresentar os benefícios desse esporte e também de expressar as percepções dos seus praticantes sobre essa atividade física, o que contribui para difundir essa modalidade pelo país, a fim de que mais pessoas conheçam, tenham acesso e pratiquem a paracanoagem. No entanto, a amostragem reduzida do estudo dificulta que esses resultados possam ser extrapolados, fazendo-se necessário a realização de novas pesquisas, com amostragem maior, que permitam uma análise mais pormenorizada da população estudada.

\section{REFERÊNCIAS}

ADELMAN, Miriam. Mulheres atletas: re-significações da corporalidade feminina. Revista Estudos Feministas, [s.1.], v. 11, n. 2, p. 445-465, dez. 2003. FapUNIFESP (SciELO). http://dx.doi.org/10.1590/s0104-026x2003000200006.

ALBARELLO, Rafael Antônio. Efeito do treinamento físico/técnico sobre a composição corporal e capacidades físicas específicas de atletas da modalidade de paracanoagem. 2014. $22 \mathrm{f}$. Monografia 
(Especialização em Educação Física), Programa de Pós-graduação em Educação Física, Universidade Federal de Santa Maria, Santa Maria, 2014.

ALMEIDA, Marco Antonio Bettine de; GUTIERREZ, Gustavo Luis; MARQUES, Renato Francisco Rodrigues. Qualidade de vida: definição, conceitos e interfaces com outras áreas de pesquisa. São Paulo: Universidade de São Paulo, 2012.

AROL, Pervin; EROğLU, Kolayiş Ipek. The effects of 8 week balance training on the kayaking performance of the beginners. Pedagogics, Psychology, Medical-biological Problems Of Physical Training And Sports, [s.1.], v. 22, n. 4, p. 170, 30 ago. 2018. Kharkov National Pedagogical University. http://dx.doi.org/10.15561/18189172.2018.0401.

BARDIN, Laurence. Análise de conteúdo. 3. ed. Lisboa: Edições 70, 2011.

BJERKEFORS, Anna; THORSTENSSON, Alf. Effects of Kayak Ergometer Training on Motor Performance in Paraplegics. International Journal Of Sports Medicine, [s.1.], v. 27, n. 10, p. 824-829, out. 2006. Georg Thieme Verlag KG. http://dx.doi.org/10.1055/s-2005-872970.

BRAZUNA, Melissa Rodrigues; CASTRO, Eliane Mauerberg de. A Trajetória do Atleta Portador de Deficiência Física no Esporte Adaptado de Rendimento: uma revisão de literatura. Revista Motriz, Rio Claro, v. 7, n. 2, p. 115-123, 2001.

BUFFART, Laurien M.; WESTENDORP, Tessa; BERG-EMONS, Rita J. van Den; STAM, Henk J.; ROEBROECK, Marij E. Perceived barriers to and facilitators of physical activity in young adults with childhood-onset physical disabilities. Journal Of Rehabilitation Medicine, [s.l.], v. 41, n. 11, p. 881-885, 2009. Acta Dermato-Venereologica. http://dx.doi.org/10.2340/16501977-0420.

CAMPOS, Marcelo Ferraz de; RIBEIRO, André Tosta; LISTIK, Sérgio; PEREIRA, Clemente Augusto de Brito; ANDRADE SOBRINHO, Jozias de; RAPOPORT, Abrão. Epidemiologia do traumatismo da coluna vertebral. Revista do Colégio Brasileiro de Cirurgiões, [s.1.], v. 35, n. 2, p. 88-93, abr. 2008. FapUNIFESP (SciELO). http://dx.doi.org/10.1590/s0100-69912008000200005.

CARDOSO, Vinícius Denardin. A reabilitação de pessoas com deficiência através do desporto adaptado. Revista Brasileira de Ciências do Esporte (Impresso), [S.L.], v. 33, n. 2, p. 529-539, jun. 2011. FapUNIFESP (SciELO). http://dx.doi.org/10.1590/s0101-32892011000200017.

CARMONA, Eduardo Klein; PEREIRA, Ester Liberato; MAZO, Janice Zarpellon. Tênis de mesa adaptado: pelos caminhos do esporte em uma cadeira de rodas. Revista Biomotriz, Cruz Alta, v. 9, n. 1, p. 38-53, 2015.

CASTRO, Eliane Mauerberg de. Atividade Física Adaptada. Ribeirão Preto: Tecmedd, 2005.

CASTRO, Flávio de Souza; CARNEIRO, Luís Marcelo. Cinemática da canoagem: revisão. Revista Brasileira de Ciência e Movimento, v. 17, n. 3, p. 114-122, 2010.

CELESTINO, Tadeu; PEREIRA, Antonino. Orientação Adaptada: Características e Potencialidades de um Desporto de Inclusão. Revista Desporto e Atividade Física para Todos - Revista Científica da FPDD, [S.L.], v. 1, n. 1, p. 12-17, 2015.

COLVER, Allan. Measuring quality of life in studies of disabled children. Paediatrics and Child Health, v.18, n.9, p.423-426, 2008. Doi: 10.1016/j.paed.2008.05.011

COMERLATO, Luciano. Os benefícios físicos, mentais e sociais da canoagem para portadores de deficiência física locomotora. Caxias do Sul: UCS, 2003. Disponível em: 
http://canoagem.org.br/arquivos/biblioteca/biblioteca canoagem deficientes fisicos 2003 luciano merlato. pdf Acesso em: 24, jun. 2020.

COSTA E SILVA, Anselmo de Athayde; MARQUES, Renato Francisco Rodrigues; PENA, Luis Gustavo de Souza; MOLCHANSKY, Sheila; BORGES, Mariane; CAMPOS, Luis Felipe Castelli Correia de; ARAðJO, Paulo Ferreira de; BORIN, João Paulo; GORLA, José Irineu. Esporte adaptado: abordagem sobre os fatores que influenciam a prática do esporte coletivo em cadeira de rodas. Revista Brasileira de Educação Física e Esporte, [s.1.], v. 27, n. 4, p. 679-687, dez. 2013. FapUNIFESP (SciELO). http://dx.doi.org/10.1590/s1807$\underline{55092013005000010 .}$.

DIAZ, Robert et al. Impact of Adaptive Sports Participation on Quality of Life. Sports Medicine and Arthroscopy Review, v.27, n.2, p.73-82, 2019. Doi: 10.1097/JSA.0000000000000242

EPHIPHANIO, Erika Hofling et al. O esporte como ferramenta de desenvolvimento para pessoas com deficiência: ações em um grupo esportivo no vale do são francisco. Revista de Extensão da Univasf, Petrolina, v. 6, n. 1, p. 97-102, 2018.

FERREIRA, Joel Saraiva; DIETTRICH, Sandra Helena Correia; PEDRO, Danielly Amado. Influência da prática de atividade física sobre a qualidade de vida de usuários do SUS. Saúde em Debate, [s.1.], v. 39, n. 106, p. 792-801, set. 2015. FapUNIFESP (SciELO). http://dx.doi.org/10.1590/0103-1104201510600030019.

FLORES, Lucinar Jupir Forner et al. Avaliação da potência aeróbia de praticantes de Rugby em Cadeira de Rodas através de um teste de quadra. Motriz: Revista de Educação Física, v. 19, n. 2, p. 368-377, 2013. http://dx.doi.org/10.1590/s1980-65742013000200014

FONTELLES, Mauro José et al. Metodologia da pesquisa científica: diretrizes para a elaboração de um protocolo de pesquisa. Revista Paraense de Medicina, [s.1.], v. 23, n. 3, p. 1-8, 2009.

GOODWIN, Donna et al. It's Okay to Be a Quad: wheelchair rugby players sense of community. Adapted Physical Activity Quarterly, v. 26, n. 2, p. 102-117, 2009. http://dx.doi.org/10.1123/apaq.26.2.102

GORGATTI, Márcia Greguol; COSTA, Roberto Fernandes da. Atividade física adaptada. Barueri: Manole, 2005.

HÄFELE, César Augusto; FREITAS, Matheus Pintanel; SILVA, Marcelo Cozzensa da; ROMBALDI, Airton José. Are physical activity levels associated with better health outcomes in people with epilepsy? Epilepsy \& Behavior, [s.1.], v. 72, p. 28-34, jul. 2017. Elsevier BV.

http://dx.doi.org/10.1016/j.yebeh.2017.04.038.

HALSON, Shona L.. Sleep in Elite Athletes and Nutritional Interventions to Enhance Sleep. Sports Medicine, [s.1.], v. 44, n. 1, p. 13-23, maio 2014. Springer Science and Business Media LLC. http://dx.doi.org/10.1007/s40279-014-0147-0.

INTERNATIONAL CANOE FEDERATION - ICF. Classification manual and form for classifiers. 2015b. Disponível em: <http://www.canoeicf.com/classification>. Acesso em 12 de janeiro de 2020.

JACOBS, Patrick L.; NASH, Mark S.; RUSINOWSKI, Joseph W.. Circuit training provides cardiorespiratory and strength benefits in persons with paraplegia. Medicine And Science In Sports And Exercise, [s.1.], p. 711-717, maio 2001. Ovid Technologies (Wolters Kluwer Health). http://dx.doi.org/10.1097/00005768-200105000-00005.

JEE, Yong-Seok. Participating in the Paralympic Games through exercise rehabilitation for individuals with disabilities. Journal of Exercise Rehabilitation, v.14 n.,2, p.158-159, 2018. Doi:10.12965/jer.1836188.094 
KAPSAL, Nathanial J et al. Effects of Physical Activity on the Physical and Psychosocial Health of Youth With Intellectual Disabilities: A Systematic Review and Meta-Analysis. J Phys Act Health, v.16, n.12, p.1187-1195, 2019. Doi: 10.1123/jpah.2018-0675.

KIM, Dong-Il et al. Effects of a 6-Week Indoor Hand-Bike Exercise Program on Health and Fitness Levels in People With Spinal Cord Injury: a randomized controlled trial study. Archives Of Physical Medicine And Rehabilitation, v. 96, n. 11, p. 2033-2040.e1, 2015.. http://dx.doi.org/10.1016/i.apmr.2015.07.010.

MANNS, Patricia J.; CHAD, Karen E.. Determining the relation between quality of life, handicap, fitness, and physical activity for persons with spinal cord injury. Archives Of Physical Medicine And

Rehabilitation, [s.1.], v. 80, n. 12, p. 1566-1571, dez. 1999. Elsevier BV. http://dx.doi.org/10.1016/s00039993(99)90331-3.

Marques, U. (1997). A exclusão social e a actividade física. In A. Marques, A. Prista, A. Junior, (Eds). Educação Física: Contexto e Inovação - Actas do V Congresso de Educação Física e Ciências do Desporto dos Países de Língua Portuguesa. (Vol.1), pp 161 - 176.

MARTINS, Paulo José Forcina; MELLO, Marco Túlio de; TUFIK, Sergio. Exercício e sono. Revista Brasileira de Medicina do Esporte, [S.L.], v. 7, n. 1, p. 28-36, 2001. http://dx.doi.org/10.1590/s1517$\underline{86922001000100006}$.

MATHERI, Joseph Mwangi; FRANTZ, Jose M. Physical activity levels among young people with physical disabilities in selected high schools in Kenya and their perceived barriers and facilitators to participation. $\mathbf{J}$ Commun Health Sci, [s.1.], v. 4, n. 1, p. 6-21, 2009.

MICHAEL, Jacob S et al. The metabolic demands of kayaking: A review. Journal of Sports Science \& Medicine, v. 7, n. 1, p. 1, 2008.

MILOSEVIC, Matija; GAGNON, Dany H.; GOURDOU, Philippe; NAKAZAWA, Kimitaka. Postural regulatory strategies during quiet sitting are affected in individuals with thoracic spinal cord injury. Gait $\&$ Posture, [s.1.], v. 58, p. 446-452, out. 2017. Elsevier BV. http://dx.doi.org/10.1016/i.gaitpost.2017.08.032.

NAHAS, Markus Vinicius. Atividade física, saúde e qualidade de vida: conceitos e sugestões para um estilo de vida ativo. 4. ed. Londrina: Midriograf, 2006.

NOREAU, Luc; SHEPHARD, Roy J.; SIMARD, Clermont; PARÉ, Giles; POMERLEAU, Pierre. Relationship of impairment and functional ability to habitual activity and fitness following spinal cord injury. International Journal of Rehabilitation Research, [s.1.], v. 16, n. 4, p. 265-276, dez. 1993. Ovid Technologies (Wolters Kluwer Health). http://dx.doi.org/10.1097/00004356-199312000-00002.

OLIVEIRA, Gilberto; CHEREM, Eduardo H.L.; TUBINO, Manoel J.G.. A inserção histórica da mulher no esporte. Revista Brasileira de Ciência e Movimento, [S.L.], v. 16, n. 2, p. 117-125, 2008.

ROPKE, Lucilene Maria; SOUZA, Amanda Gouvea; BERTOZ, André Pinheiro de Magalhães; ADRIAZOLA, Manuel Martin; ORTOLAN, Erika Verusca Paiva; MARTINS, Regina Helena; LOPES, Weder Carneiro; RODRIGUES, Cesar Diogo Benichio; BIGLIAZZI, Renato; WEBER, Silke Anna Theresa. Efeito da atividade física na qualidade do sono e qualidade de vida: revisão sistematizada. Archives Of Health Investigation, [s.1.], v. 6, n. 12, p. 561-566, 29 jan. 2018. Archives of Health Investigation. http://dx.doi.org/10.21270/archi.v6i12.2258.

SAMPIERI, Roberto Hernandes; COLLADO, Carlos Fernández; LUCIO, María del Pilar Baptista. Metodologia da Pesquisa. 5. ed. Porto Alegre: Penso, 2013. 
SCHREIBER, Roberto; SOUZA, Cristiane M; PAIM Layde R, et al. Impact of regular physical activity on adipocytokines and cardiovascular characteristics in spinal cord injured subjects, Archives of Physical Medicine and Rehabilitation, v.99, n.8, p. 1561-1567, 2018. Doi: 10.1016/ j.apmr.2018.02.010.

SKUCAS, Kestutis; POKVYTYTE, Vaida. Combined strength exercises on dry land and in the water to improve swimming parameters of athletes with paraplegia. The Journal Of Sports Medicine And Physical Fitness, [S.L.], v. 58, n. 3, p. 197-203, fev. 2018. Edizioni Minerva Medica S.p.A..

http://dx.doi.org/10.23736/S0022-4707.16.06702-5.

TAYLOR, Leah Peri Siegel; MCGRUDER, Juli Eyans. The Meaning of Sea Kayaking for Persons With Spinal Cord Injuries. American Journal Of Occupational Therapy, [s.1.], v. 50, n. 1, p. 39-46, 1 jan. 1996. AOTA Press. http://dx.doi.org/10.5014/ajot.50.1.39.

THE WHOQOL GROUP. The World Health Organization quality of life assessment (WHOQOL): position paper from the world health organization. Social Science \& Medicine, [s.1.], v. 41, n. 10, p. 14031409, nov. 1995. Elsevier BV. http://dx.doi.org/10.1016/0277-9536(95)00112-k.

TONON, Luciane Maria Micheletti. A história de vida da nadadora Susana Schnarndorf. In: Anais do XIII Encontro Nacional de História Oral - História Oral, Práticas Educacionais e Interdisciplinaridade. 2016. p. 1-11. 15 .

ZOPPI, Cláudio Cesar et al. Physiological and Performance Improvements during a Training Season in Paralympic Rowers. Journal Of Exercise Physiology Online, [S.L.], v. 17, n. 3, p. 88-101, June 2014. Acessado em 30 de setembro de 2020.

\section{NOTAS DE AUTOR}

\section{AGRADECIMENTOS}

Confederação Brasileira de Canoagem pelo apoio no desenvolvimento da paracanoagem na região de Petrolina - Pernambuco.

\section{CONTRIBUIÇÃO DE AUTORIA}

Concepção do manuscrito: D.S. Araújo, S.S. Lima, L.G.T. Costa

Coleta de dados: D.S. Araújo, L.G.T. Costa

Análise de dados: D.S. Araújo, S.S. Lima

Discussão dos resultados: R.C. Vanali, L.A.M. Santos

Produção do texto: D.S. Araújo, S.S. Lima; L.G.T. Costa

Revisão e aprovação: R.C. Vanali, L.A.M. Santos, L.G.T. Costa

\section{FINANCIAMENTO}

Proex - Pró reitoria de extensão, Universidade Federal do Vale do São Francisco - UNIVASF

CONSENTIMENTO DE USO DE IMAGEM - Não se aplica.

\section{APROVAÇÃO DE COMITÊ DE ÉTICA EM PESQUISA}

Aprovado pelo comitê de ética da Universidade Federal do Vale do São Francisco - UNIVASF (parecer n. 3.892.500), na data de 02 de março de 2020.

CONFLITO DE INTERESSES - Não se aplica. 


\section{LICENÇA DE USO}

Os autores cedem à Motrivivência - ISSN 2175-8042 os direitos exclusivos de primeira publicação, com o trabalho simultaneamente licenciado sob a Licença Creative Commons Attribution Non-Comercial ShareAlike (CC BY-NC SA) 4.0 International. Esta licença permite que terceiros remixem, adaptem e criem a partir do trabalho publicado, desde que para fins não comerciais, atribuindo o devido crédito de autoria e publicação inicial neste periódico desde que adotem a mesma licença, compartilhar igual. Os autores têm autorização para assumir contratos adicionais separadamente, para distribuição não exclusiva da versão do trabalho publicada neste periódico (ex.: publicar em repositório institucional, em site pessoal, publicar uma tradução, ou como capítulo de livro), com reconhecimento de autoria e publicação inicial neste periódico, desde que para fins não comerciais e compartilhar com a mesma licença.

\section{PUBLISHER}

Universidade Federal de Santa Catarina. Programa de Pós-Graduação em Educação Física. LaboMídia - Laboratório e Observatório da Mídia Esportiva. Publicado no Portal de Periódicos

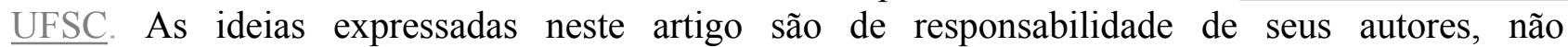
representando, necessariamente, a opinião dos editores ou da universidade.

\section{EDITORES}

Mauricio Roberto da Silva, Giovani De Lorenzi Pires, Rogério Santos Pereira.

\section{EDITORA DE SEÇÃO}

Bianca Natália Poffo.

\section{REVISÃO DO MANUSCRITO E METADADOS}

João Caetano Prates Rocha; Keli Barreto Santos.

\section{HISTÓRICO}

Recebido em: 20 de julho de 2020.

Aprovado em: 30 de outubro de 2020. 